\title{
COMMUNITY GARDENING DURING TIMES OF CRISIS: RECOMMENDATIONS FOR COMMUNITY-ENGAGED DIALOGUE, RESEARCH, AND PRAXIS
}

Authors: Angie Mejia ${ }^{1}$, Manami Bhattacharya ${ }^{2}$, Amanda Nigon-Crowley ${ }^{3}$, Kelly Kirkpatrick ${ }^{4}$, and Chandi Katoch ${ }^{5}$.

This is currently a final version of a manuscript submitted and conditionally accepted at Journal of Agriculture, Food Systems, and Community Development; a summarized version will also be available as a blog at their affiliated site, Voices from the Grassroots. Questions should be directed via email at amejiame@r.umn.edu.

\begin{abstract}
Using ongoing reflections from our recent work as members of a community gardening initiative, we outline relevant priorities for researchers, policymakers, and community practitioners to examine the role of community gardens in addressing the effects of COVID-19 on the lives of intersectionally-diverse growers. To understand how COVID-19 has influenced the practices of community-led urban agricultural spaces, we suggest future efforts take into consideration three essential areas of focus: uses of community gardening in combating food insecurity during a pandemic, changes in community garden operations as a response to crises, and community gardening's role in nurturing emotional well-being.
\end{abstract}

\section{INTRODUCTION}

How do community gardens - as spaces to grow fresh and nutritious food - socially, politically, and culturally respond to a pandemic? This essay is informed by the work of a range of stakeholders connected to The Village Community Garden and Learning Center, a community garden initiative located in Rochester, Minnesota. After introducing our project, we outline three areas of interest to growers, community organizers, students, researchers, and other stakeholders navigating COVID-19's effects on day-to-day operations. We hope that our perspectives encourage conversation among individuals and groups working in projects that intersect food, community gardening, and collective wellness. We also address challenges from the pandemic and seek to build new practices that empower our communities.

\footnotetext{
${ }^{1}$ Assistant Professor and Civic Engagement Scholar, Center for Learning Innovation, University of Minnesota Rochester.

${ }^{2}$ Division of Health Policy and Management, University of Minnesota Twin Cities.

${ }^{3}$ The Village Community Garden and Learning Center, Rochester, MN.

4 Plant.a.seed., Rochester, MN.

${ }^{5}$ Center for Learning Innovation, University of Minnesota Rochester
} 


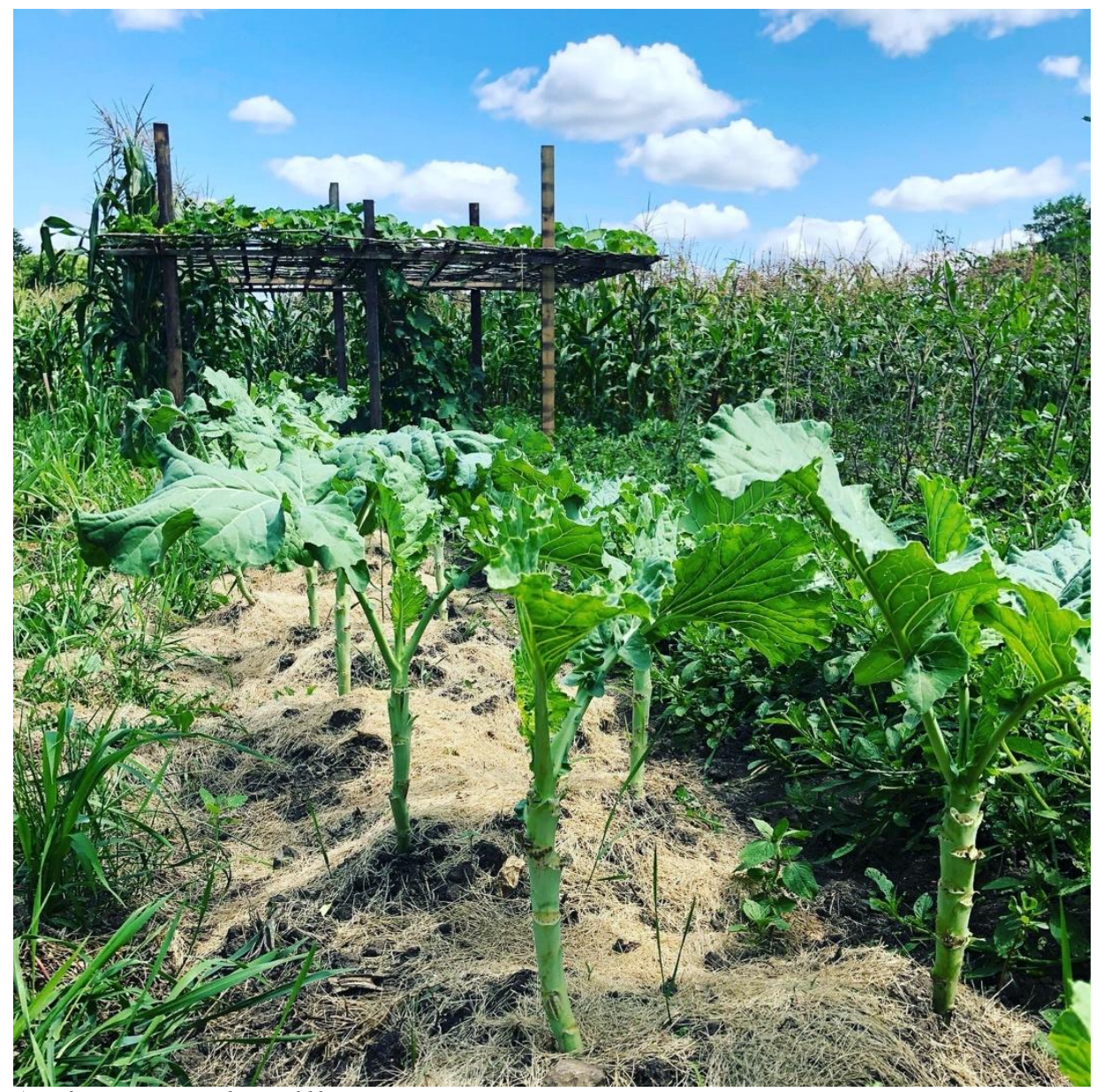

A bit about The Village

The gardeners within The Village established their need for access to land. Most of the growers live in housing where they do not have access to a garden or larger areas of land to grow commodities native to their homeland which they can then sell within their communities and at local markets. As an organization serving gardeners from marginalized communities, the Village strives to find urban growing spaces with necessary accommodations: parking and access to the city bus route, water, and proximity to a restroom. The gardeners are assigned their own plot to manage for the growing season at no cost (unless they can donate to help with expenses). The organization's steering board seeks local funding and grants to support operations. The gardeners show a willingness to work together on special projects and with the coordinators in managing aspects of the garden. All of the work conducted and produced by The Village (such as this essay) centers on the intersectionally-rich perspectives of our growers (totaling over 130 heads of households, with over $90 \%$ representing non-European, non-Anglo ethnic/racial groups), a steering board (whose membership is around 50\% non-European White), as well as the garden's leadership, comprised of an academic PI and community co-PI representing the two largest communities of growers in the area-Mexican and Cambodian, respectively. 


\section{A COMMUNITY GARDEN AT A TIME OF CRISIS}

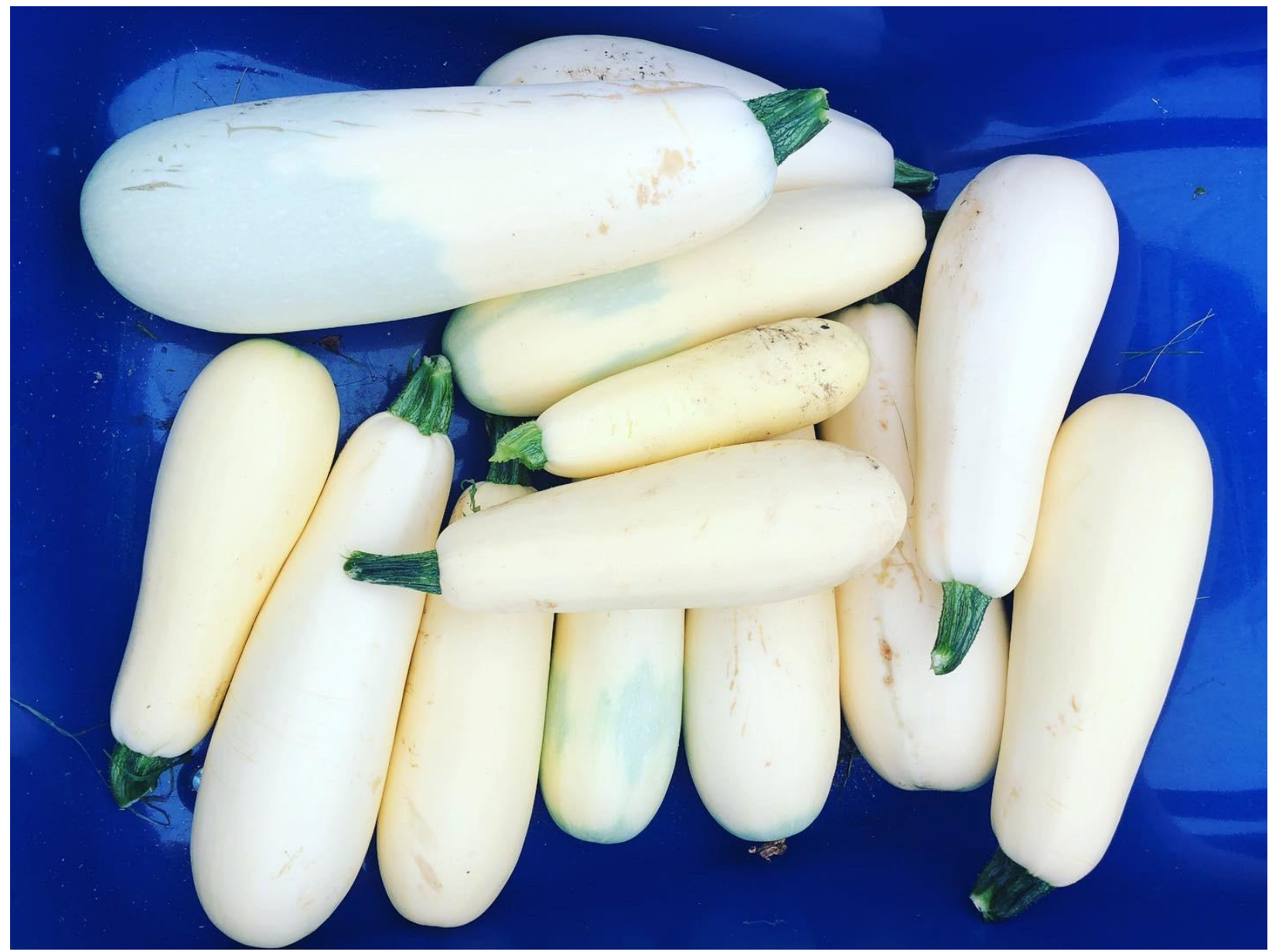

Our ongoing conversations as board members, growers, and activist-scholars have highlighted possible issues for further exploration by those looking at the role of community gardens during pandemic times. These spaces can not only provide access to fresh food but also supporting the collective well-being of racially/ethnic minoritized groups and individuals navigating moments of crisis. While these insights are not representative of an agenda that would benefit a more mainstream community gardening audience, we see the importance of our perspectives as highlighting broader social inequities arising from the pandemic, as well as the role of community gardens as possible spaces of social transformation.

\section{The Role of Community Growing Spaces to Grow and Supplement Food During the Pandemic}

Community gardens and other spaces can help families weather pandemic-related economic losses by supplementing nutritious foods ( $\mathrm{Lal}, 2020)$. In light of the ongoing crisis, what are community gardens' roles in the experience and possible alleviation of individuals' and groups' food insecurity? Currently, families respond to bouts of pandemic-triggered food insecurity by spending less on groceries, with more than one-third of these families having difficulties affording food in addition to other basic needs(Gonzalez et al., 2020). Since 
community gardening has been one of many strategies used by people to supplement much needed nutritious food, those working in similar initiatives are well-positioned to explore how different community garden growers address food insecurity emerging from COVID-19 induced economic issues.

Although the adjustment of supply chain practices has mitigated the bare shelves we saw early in the pandemic, maintaining culturally-specific food can be difficult for communities of color and immigrants during moments of crisis (Aronson, 2014). Due to employee sickness, store closure, or supply interruptions, small businesses that sell "specialty" food items essential to culturally-specific habits are especially at risk. Specialty crops, shipping from other countries, and additional quarantine requirements have limited supplies and increased the cost of these foods (Gray, 2020). Furthermore, immigrants use community gardening to maintain social and food traditions (Hartwig \& Mason, 2016). Our community board members have indicated growers' worries about their ability to access culturally-specific produce if grocery stores were to close during this or the next wave of this pandemic. Researchers and practitioners alike should also examine how community gardening, on a larger scale, may help both gardeners and the greater community supplement culturally diverse foods during COVID-19 interruptions.

\section{Community Gardens: Pivoting and Responding to COVID-19}

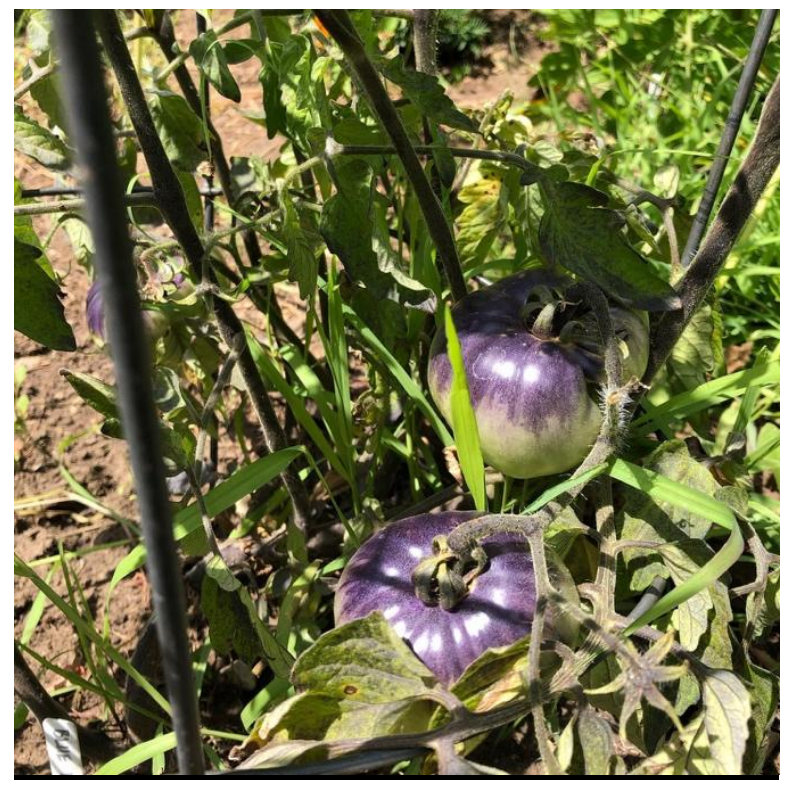

The Village has responded to COVID-19 in different ways. During the 2019 growing season, the Village's garden plots were assigned at about $75 \%$ capacity but only utilized at about $65 \%$, leaving an acre of land without food production. During the 2020 growing season, which coincided with the pandemic's beginning, the plots were at $100 \%$ capacity, with additional growers wait-listed. We also had to develop safe, social-distancing protocols that were linguistically and culturally relevant to our growers. The Village's steering board strategized to allocate additional growing space as the number of gardeners increased due to a food pantry's diminished capacity and an increase of people seeking space to grow. 
Growers state that the community garden supplements their food supply and provides places to grow produce from their native countries. Also, several of our growers have asked for increased capacity to grow for their families and to sell in local markets, while others are looking for ways to raise livestock. Many of the long-term growers have stated that the community garden, as a whole, looks better than it has in the past. Our steering committee is looking for ways to further support our gardeners as they navigate the pandemic.

Community gardens (and the desire to grow one's food) have been blossoming over the last decade. Still, the pandemic may have advanced this desire so significantly that most seed companies temporarily stopped taking orders to catch up with demand. Locally, this occurred at our Seed Library at the Rochester Public Library, and regionally at seed houses within 100 miles of Rochester. To meet the increased demand for growing food after Minneapolis' calls for justice in the wake of George Floyd's death, in addition to COVID-19, newly created organizations sought donations to supply community gardens with seeds and vegetable transplants in foodinsecure and low-income areas. While some growers were starting seeds and planting, the need for community gardens, such as ours, has emerged.

Our local food bank, which serves the broader Southern Minnesota region, could not distribute fresh food and could not take fresh vegetable donations, significantly limiting access. Other food banks, such as the one serving local university students, had to change operations and work on procedures and guidelines for staff before accepting donations from The Village. This new void created a gap in services for many that relied on assistance to meet their food needs.

\section{Community Gardens as "Beyond Food" Spaces}

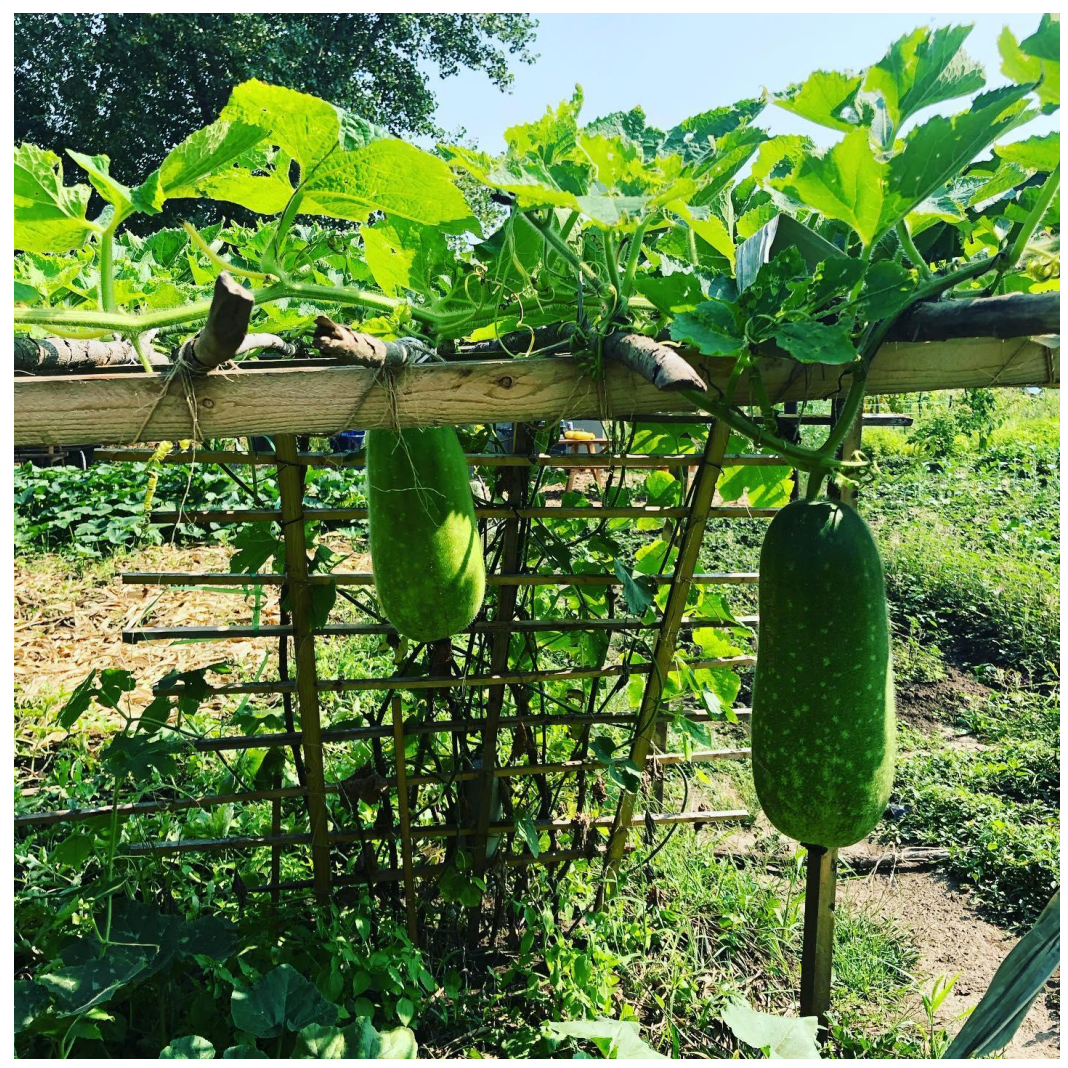


Individuals are currently experiencing a loss of connection and isolation from COVID-19 stay-at-home orders (Berg-Weger \& Morley, 2020; Vannini, 2020). Community gardening increases social capital, social support, and social connectedness (Kingsley \& Townsend, 2006). Specifically, participants see them as spaces to connect and socialize with others outside their social networks and improve their sense of cohesion. In some communities, especially after trauma, disaster, or tragedy, gardening has been used to promote healing. Examples of this include gardens established by survivors of Hurricane Katrina, refugees after immigration (Bailey, 2017), the community garden set in Christchurch, New Zealand in response to the earthquakes (Shimpo et al., 2019), and, most recently, citizens in North Minneapolis after the murder of George Floyd (Townsend, 2020). As we start working to collect qualitative and quantitative data on how our gardeners benefit from The Village, we encourage others to map out and share how growing spaces in their localities are helping various communities, both mainstream and minoritized.

Community gardens, such as The Village, continue to provide communities, especially those multiply marginalized, to maintain existing connections. Via the link to other gardenerseven when these interactions occur in a socially-distanced fashion - we have noticed that ownership of plots has provided social/emotional support during the pandemic. Our ongoing ethnographic forays and exploratory interviews with gardeners show that The Village has provided much-needed space for well-being during great stress. First, The Village has become a place that offers gardeners a healthy social activity during COVID-19, while so many people have idle time due to unemployment or being furloughed. Also, the garden has allowed for the strengthening of social relationships between new and existing gardeners. Finally, we have also observed that our community gardeners have benefited from the support of the coordinators, volunteers, and members of the steering committee.

\section{Growing Together}




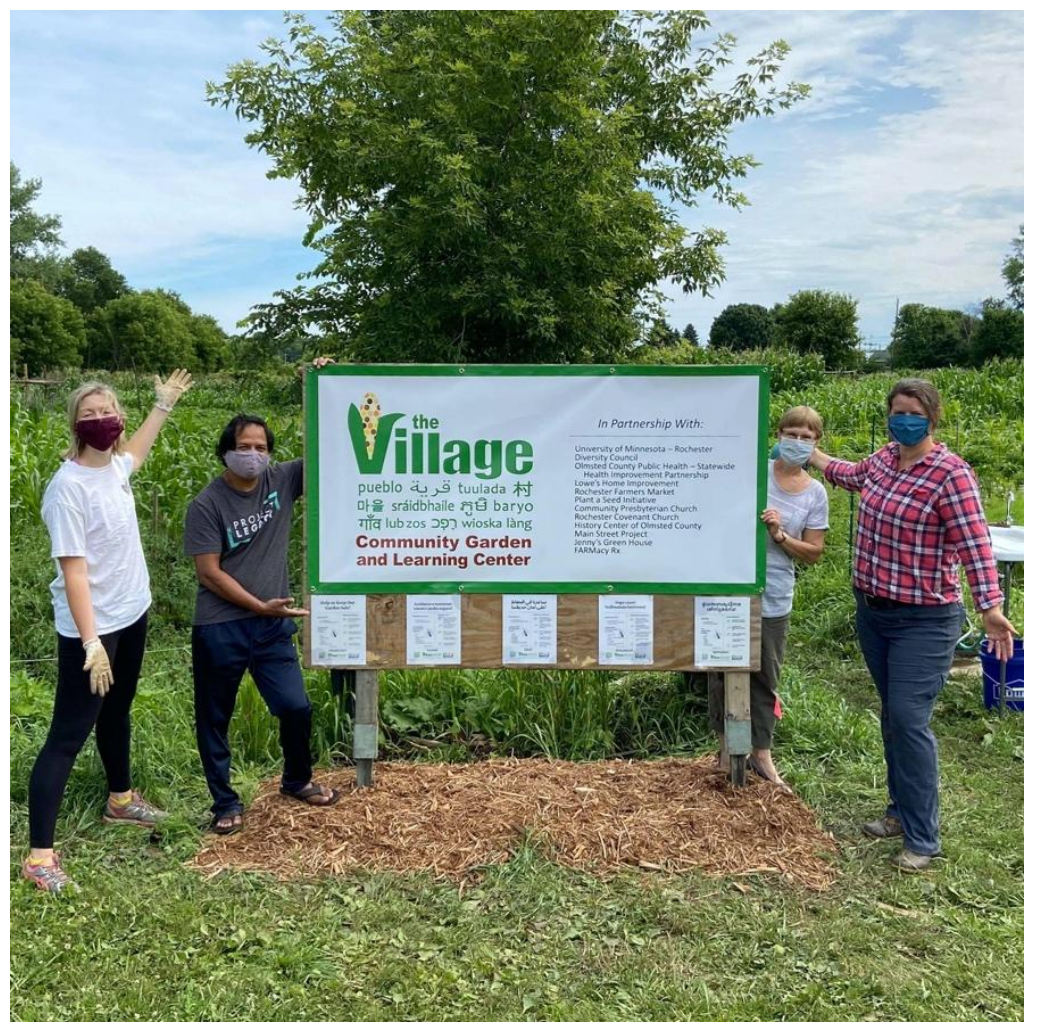

In this essay, we outlined three areas of interest to researchers, practitioners, and other stakeholders working in small-scale agricultural initiatives as they respond to the pandemic's effects on their communities. In highlighting possible points of exploration that address the ongoing challenges community gardening projects face from COVID-19, we seek to stimulate conversations on these spaces' role for minoritized communities as similar health crises threaten their relationship to food. COVID-19's unpredictable effects do not make it impossible to plan and map how community gardens, and similar-positioned initiatives might creatively respond to issues of access, consumption, and the role of food and small-scale growing initiatives in pandemic times. In closing, we propose that food and community are essential nexuses for building new social justice practices and envisioning a new normal. In light of the barriers and pressures experienced by our community gardens during crises, we are inspired by the possibilities that these sites can nurture transformative visions that go beyond resignation to a "new normal" into developing ways of building stronger community bonds through collective growing spaces.

\section{Cited Sources}

Aronson, R. A. (2014). Eating in Crisis: Culturally Appropriate Food and the Local Food Movement in the Lives of Domestic Violence Survivors. 
Bailey, P. (2017, August 10). Community Gardening a Boon to Neighborhoods in Crisis. Non Profit News | Nonprofit Quarterly. https://nonprofitquarterly.org/community-gardeningboon-neighborhoods-crisis/

Berg-Weger, M., \& Morley, J. (2020). Loneliness and social isolation in older adults during the Covid-19 pandemic: Implications for gerontological social work.

Gonzalez, D., Karpman, M., Kenney, G., \& Zuckerman, S. (2020). Hispanic Adults in Families with Noncitizens Disproportionately Feel the Economic Fallout from COVID-19 (Health Policy Center) [Brief]. Urban Institute.

Gray, R. S. (2020). Agriculture, transportation, and the COVID-19 crisis. Canadian Journal of Agricultural Economics/Revue Canadienne d'agroeconomie.

Hartwig, K. A., \& Mason, M. (2016). Community gardens for refugee and immigrant communities as a means of health promotion. Journal of Community Health, 41(6), 1153-1159. https://doi.org/10.1007/s10900-016-0195-5

Kingsley, J., \& Townsend, M. (2006). 'Dig in'to social capital: Community gardens as mechanisms for growing urban social connectedness. Urban Policy and Research, 24(4), 525-537.

Lal, R. (2020). Home gardening and urban agriculture for advancing food and nutritional security in response to the COVID-19 pandemic. Food Security, 1-6.

Shimpo, N., Wesener, A., \& McWilliam, W. (2019). How community gardens may contribute to community resilience following an earthquake. Urban Forestry \& Urban Greening, 38, 124-132. https://doi.org/10.1016/j.ufug.2018.12.002 
Townsend, M. (2020, June). North siders work the land as an act of healing. MPR News. https://www.mprnews.org/story/2020/06/22/waiting-for-justice-for-floyd-north-siderswork-the-land-as-an-act-of-healing

Vannini, P. (2020). COVID-19 as Atmospheric Dis-ease: Attuning into Ordinary Effects of Collective Quarantine and Isolation. Space and Culture, 23(3), 269-273.

https://doi.org/10.1177/1206331220938640 